Research Article

\title{
Laboratory Study of Flocculation and Pressure Filtration Dewatering of Waste Slurry
}

\author{
Fanlu Min $\mathbb{D}^{1},{ }^{1,2}$ Dengfeng Wang $\mathbb{D}^{2},{ }^{2}$ Jiarui Du, ${ }^{2}$ Hangbiao Song, ${ }^{3}$ Yucheng Wang, ${ }^{2}$ \\ Huanjie $\mathrm{Lv}^{2}$ and Jiawei $\mathrm{Ma}^{2}$ \\ ${ }^{1}$ Key Laboratory of Geomechanics and Embankment Engineering, Hohai University, Ministry of Education, \\ Nanjing 210098, China \\ ${ }^{2}$ College of Civil and Transportation Engineering, Hohai University, Nanjing 210098, China \\ ${ }^{3}$ UEE Research Unit-Geomechanics and Engineering Geology, University of Liège, Liège 4000, Belgium
}

Correspondence should be addressed to Dengfeng Wang; 181604010055@hhu.edu.cn

Received 21 October 2019; Revised 1 September 2020; Accepted 5 September 2020; Published 30 September 2020

Academic Editor: Paolo Castaldo

Copyright (c) 2020 Fanlu Min et al. This is an open access article distributed under the Creative Commons Attribution License, which permits unrestricted use, distribution, and reproduction in any medium, provided the original work is properly cited.

In the process of large-scale urban construction, large amounts of waste slurry are produced. The slurry has a high water content and is difficult to precipitate naturally, resulting in low treatment efficiency. To improve the treatment efficiency of slurry, a variety of inorganic and organic polymer flocculants were used to carry out flocculation settlement tests on the slurry. The changes in the slurry properties and the filtration dewatering effect after flocculation were tested. The results show that the addition of flocculant makes the slurry particles form aggregates, which leads to rapid precipitation of the slurry. The use of an inorganic flocculant significantly reduced the zeta potential of the slurry. Organic polymer flocculant, however, had little effect on the slurry potential, but did cause the slurry to produce larger size aggregates, resulting in a better flocculation effect than inorganic flocculant. Inorganic flocculants and organic flocculants can improve the pressure filtration dewatering performance of slurry. CPAM12 (cationic polyacrylamide, with a relative molecular weight of 12 million Daltons) had the best overall effect. The formation of aggregates after flocculation and the change in the nonuniformity coefficient $\left(C_{u}\right)$ were the main cause of improvement of pressure filtration dewatering performance of the slurry. When $C_{u}$ decreases from 11.85 to 8.75 , the time required for pressure filtration stabilization is shortened by $70 \%$. The nonuniformity coefficient of flocculated slurry can be used to evaluate flocculants, determine the optimal dosage, and predict the dewatering effect.

\section{Introduction}

Slurry is widely used in large-scale infrastructure construction in China because of its excellent wall protection and slag carrying characteristics, in addition to other advantages, such as its low cost and ready availability $[1,2]$. However, large amounts of waste slurry are unavoidably produced in the construction process. The slurry has a high water content, a very large volume, and a need to occupy a large amount of land for settlement treatment. Long time of settlement is often required. Only when the water content of slurry drops to a specified level can subsequent treatment be carried out (e.g., vacuum preloading [3]). This not only wastes a lot of land resources but also causes secondary pollution to the environment. Therefore, the rapid separation of slurry and water has become a major challenge in the engineering field.

Historically, geotextile bag method [4-6] and vacuum filtration $[7,8]$ are commonly used for separation of slurry and water. In geotextile bag dewatering, slurry is pumped into a geotextile bag, and the weight of the slurry is used to filter out water and retain soil particles in the geotextile bag. In vacuum suction filtration dewatering, a vacuum suction filter tube is installed in the slurry to accelerate the dewatering through negative pressure. The biggest problem with these two methods is that it is prone to blockage [7-12], which leads to a reduction in the dewatering efficiency. To address the problem of geotextile blockages, engineers and 
technicians periodically apply pressure and suction to the bottom slurry filter tube, which reduces the blockage, but does not significantly increase the drainage efficiency [7, 13]. In the field of municipal sludge dewatering, mechanical dewatering methods, such as the plate-frame filter press [14] and belt filter press [15], are widely used. To improve the dewatering efficiency, flocculants, such as polyacrylamide [16] and polyaluminium chloride [17], are added to pretreat the sludge, which can reduce the loss of fine particles [18] and accelerate the dewatering time [19-22].

Currently, waste slurry treatment is guided by knowledge from the sludge dewatering field. First, flocculant is added to flocculate and concentrate the slurry, and then the concentrated slurry is dewatered by mechanical pressure filtration. In the process of slurry treatment, flocculant and pressure filtration are a continuous and integrated process. Flocculation changes the properties of slurry and further affects its pressure filtration dewatering performance [18-22]. Therefore, the selection of flocculants and its dosage are particularly important. However, most researchers only select the flocculant and its dosage according to the effect of slurry flocculation and settlement, ignoring its influence on the pressure filtration dewatering performance of slurry [23-25], which is not inclusive.

The present study investigated the treatment of engineering waste slurry placed in the Nanjing Slurry Comprehensive Disposal Center. A flocculation settling test was carried out using various inorganic and organic flocculants, and the changes in the properties of the slurry after flocculation and the effect of pressure filtration dewatering, were tested. From the basic properties of the flocculated slurry, the reasons for the difference of flocculation effect of different flocculants and the potential mechanism of flocculants affecting pressure filtration dewatering performance of slurry are discussed. Through the above discussions, a flocculant selection method based on flocculation effect and pressure filtration dewatering effect is attempted to find out. The results of the present study provide a reference for the treatment of high-water-content engineering waste slurry.

\section{Materials and Methods}

\subsection{Materials}

2.1.1. Slurry. Waste slurry stored in the Nanjing Comprehensive Disposal Center was used in this investigation. The basic physical properties of the slurry were determined according to the Standard of the Geotechnical Test Method (GB/T 50123-1999) (see Table 1). The water content of the slurry was as high as $900 \%$ (water content is defined as the ratio of water mass to the dry matter mass). The particle size distribution curve of the slurry was measured using a laser particle size analyzer (Malvern Mastersizer 2000). Figure 1 shows grain size distribution of the slurry. It can be seen that the slurry particle size is small, and the proportion of particles smaller than $75 \mu \mathrm{m}$ is as high as $86 \%$.

2.1.2. Flocculants. Five kinds of flocculants were used in this experiment. Two were inorganic and three were organic polymers. The inorganic flocculants used were ferric chloride $\left(\mathrm{FeCl}_{3}\right)$ and polyaluminium chloride (PAC). The organic polymer flocculants used were cationic polyacrylamide with a relative molecular weight of 3 million (CPAM3), cationic polyacrylamide with a relative molecular weight of 12 million (CPAM12), and anionic polyacrylamide with a relative molecular weight of 12 million (APAM12). The flocculants should be used in the solution form. Suitable hydrolysis concentrations and dosage ranges for the flocculants used in the present study were known from some literatures [16, 17, 23-25]. The hydrolysis concentration of the flocculants used and the five dosages of each flocculant are given in Table 2. The solution concentration refers to the mass concentration. The dosage of flocculants added was based on the dry weight of the soil in the slurry. For example, $0.1 \%$ refers to a flocculating solution prepared from $0.1 \mathrm{~g}$ PAM that was added to $1000 \mathrm{~g}$ slurry with a water content of $900 \%$.

\subsection{Methods}

2.2.1. Flocculation Settlement Test. $1200 \mathrm{ml}$ of slurry was put into a beaker, stirred for 3 minutes at a speed of $200 \mathrm{r} / \mathrm{min}$ to ensure uniformity. Then, the prepared flocculant solution was added to the slurry, stirred for 1 minute at a speed of $100 \mathrm{r} / \mathrm{min}$ and then quickly poured into a measuring cylinder until the $1000 \mathrm{ml}$ scale line was reached. The slurry was then allowed to settle naturally for 7 hours (Figure 2).

Recording the readings of the measuring cylinder every 5 minutes, the water content of slurry at a given time can be estimated. The calculation process is as follows.

Firstly, the solid particle mass $\left(m_{0}\right)$ of slurry in the beaker before adding flocculant solution is calculated using the following equation:

$$
m_{0}=\frac{V_{0} \rho}{1+w_{0}},
$$

where $V_{0}(1200 \mathrm{ml})$ is the volume of the slurry sample in the beaker, $w_{0}(900 \%)$ is the initial water content of slurry, and $\rho$ $\left(1030 \mathrm{~kg} / \mathrm{m}^{3}\right)$ is the density of the slurry sample in the beaker.

The quality $\left(m_{1}\right)$ of water in slurry in the beaker before adding flocculant is calculated using the following equation:

$$
m_{1}=m_{0} w_{0}
$$

The quality $\left(m_{2}\right)$ of flocculant can be calculated using the following equation:

$$
m_{2}=m_{0} n_{1} \text {, }
$$

where $n_{1}$ is the dosage of flocculant (\%).

The quality $\left(m_{3}\right)$ of water needed to prepare flocculant into solution is calculated using the following equation:

$$
m_{3}=\frac{m_{2}\left(1-n_{2}\right)}{n_{2}}
$$

where $n_{2}$ is the concentration of flocculant solution.

Because the quality of flocculant is very small, the effect of flocculant volume on flocculant solution volume is 
TABle 1: Basic properties of the slurry.

\begin{tabular}{lcccc}
\hline Water content (\%) & Density $\left(\mathrm{kg} / \mathrm{m}^{3}\right)$ & Liquid limit $(\%)$ & Plastic limit (\%) & Organic content (\%) \\
\hline 900 & 1030 & 60 & 24 & 0.42 \\
\hline
\end{tabular}

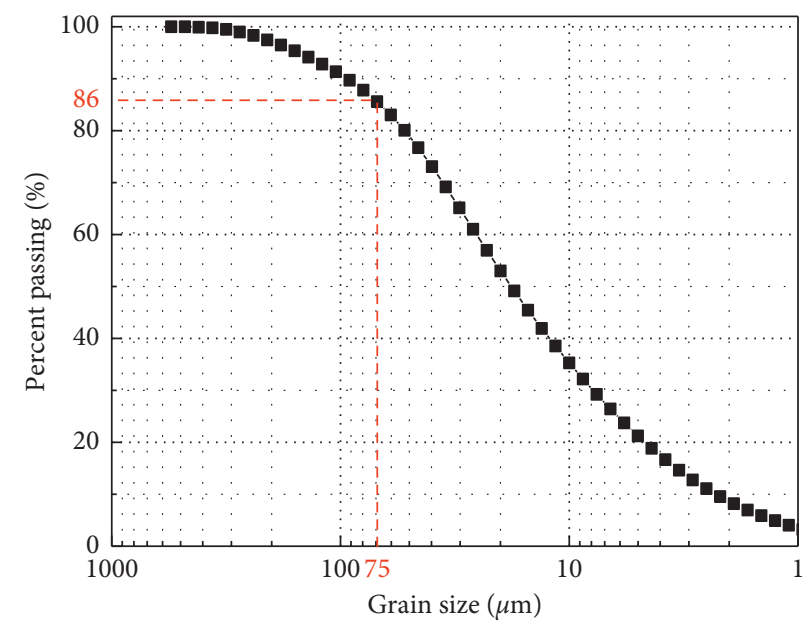

Figure 1: Grain size distribution of the slurry.

TABle 2: Flocculant types and dosage.

\begin{tabular}{lccc}
\hline Flocculant & Solution concentration (\%) & Dosage (\%) & Purity \\
\hline $\mathrm{FeCl}_{3}$ & 10 & $0.8,1.6,2.4,3.2,4$ & Analytical pure \\
$\mathrm{PAC}$ & 10 & $0.4,0.8,1.6,2.4,3.2$ & Analytical pure \\
$\mathrm{CPAM} 3$ & 0.3 & $0.1,0.2,0.3,0.5,0.7$ & Analytical pure \\
$\mathrm{CPAM} 12$ & 0.3 & $0.1,0.2,0.3,0.5,0.7$ & Analytical pure \\
$\mathrm{APAM} 12$ & 0.3 & $0.1,0.2,0.3,0.5,0.7$ & Analytical pure \\
\hline
\end{tabular}

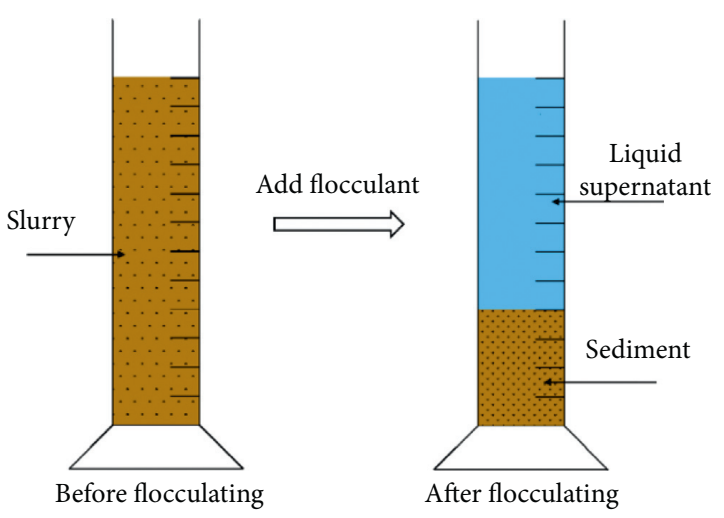

Figure 2: Schematic diagram of the flocculation settlement test.

neglected. The flocculant solution volume $V_{1}$ can be estimated using the following equation:

$$
V_{1}=\frac{m_{3}}{\rho_{1}},
$$

where $\rho_{1}\left(1000 \mathrm{~kg} / \mathrm{m}^{3}\right)$ is the water density.

Then, the quality $\left(m_{4}\right)$ of slurry solid particles in the measuring cylinder and the quality $\left(m_{5}\right)$ of water in the measuring cylinder can be estimated from (6) and (7), respectively:

$$
\begin{aligned}
& m_{4}=\frac{m_{0}}{V_{0}+V_{1}} V_{2}, \\
& m_{5}=\frac{m_{3}+m_{1}}{V_{0}+V_{1}} V_{2},
\end{aligned}
$$

where $V_{2}(1000 \mathrm{ml})$ is the volume of sample in the measuring cylinder.

Finally, the water content $(w)$ of the sediment can be estimated using the following equation according to the reading of the measuring. The influence of a small amount of solid particles remained in the liquid supernatant was ignored:

$$
w=\frac{m_{5}-\rho_{1}\left(V_{1}-h S\right)}{m_{4}},
$$

where $h(\mathrm{~cm})$ is the reading height and $S\left(\mathrm{~cm}^{2}\right)$ is the crosssectional area of the measuring cylinder.

After the flocculated slurry had settled for $1 \mathrm{~h}, 4 \mathrm{~h}$, and $7 \mathrm{~h}$, a small amount of supernatant was taken to measure the suspended solid (SS) matter content. A laser particle size analyzer and a zeta potentiometer were used to measure the size of slurry aggregates and the zeta potential. To obtain actual size data of the aggregates in the mud, the ultrasonic system of the laser particle size was closed, and a series of pump speed and sampling times were selected to optimize 
the measurement parameters [26]. The final pump speed was $1500 \mathrm{rpm}$ and the sampling interval was $5 \mathrm{~s}$ in this study. Under this experimental condition, it is reasonably assumed that the slurry and water was mixed sufficiently with no air bubbles and the break of flocculation in a short time period. Thus, the measured size of the aggregates could be used for qualitative analyses [27].

2.2.2. Pressure Filtration Dewatering Test. The slurry pressure filter device shown in Figure 3 was used to simulate the mechanical dewatering of the flocculated slurry. Mechanical dewatering uses an external force to drain the water in the slurry from the pores. The device consists of a plexiglass column, a piston, a beaker, a data acquisition device, and a pneumatic device. At the bottom of the organic glass column, gravel sand with a particle size of $2-5 \mathrm{~mm}$ was installed as the permeable layer (mainly for drainage) and was saturated by the reverse saturation method so as to avoid affecting the measurement of the mass of filtered water. Then, a layer of geotextile was laid on the top as the filter medium and then flocculated slurry (the flocculation time of slurry was $1 \mathrm{~h}$, which is determined according to existing slurry treatment process, the sample loading quality was recorded, and the water content of slurry was measured) was poured into the glass column. The piston was then installed and the flange was sealed, pressure was applied to the organic glass column, and the test was initiated. Pressure filtration lasted $2 \mathrm{~h}$. A data acquisition device which consists of only one balance was used to record the quality $\left(m_{\mathrm{t}}\right)$ of the filtered water during of slurry pressure filtration.

The water content $\left(w_{2}\right)$ of slurry at a given time in the process of slurry pressure filtration was calculated using the following equation:

$$
w_{2}=\mathrm{w}_{1}-\frac{m_{\mathrm{t}}\left(1+\mathrm{w}_{1}\right)}{\mathrm{m}_{6}},
$$

where $w_{1}$ is the water content of mud before filter pressing $(\%), m_{6}$ is the quality of the slurry sample $(\mathrm{g})$, and $m_{\mathrm{t}}$ is the balance reading of the filtered water quality at a given time (g).

\section{Results and Discussion of Flocculation Settlement Test and Basic Properties of Flocculated Slurry Test}

3.1. Result of Flocculation Settlement Test. Five types of flocculants were used to flocculate and settle the slurry. Each flocculant was applied in five dosages (see Table 2). A total of 25 groups of tests were carried out. The water content of the sediment was calculated by measuring the cylinder reading, and the water content curve of the sediment was drawn. The SS content in the supernatant at different times was measured at selected times and a SS curve was drawn. Figure 4 is the change curve of slurry water content after adding CPAM12. It can be seen that the flocculation settling speed of slurry was greatly increased when the dosage of CPAM12 is added from $0.1 \%$ to $0.2 \%$, and the water content of the

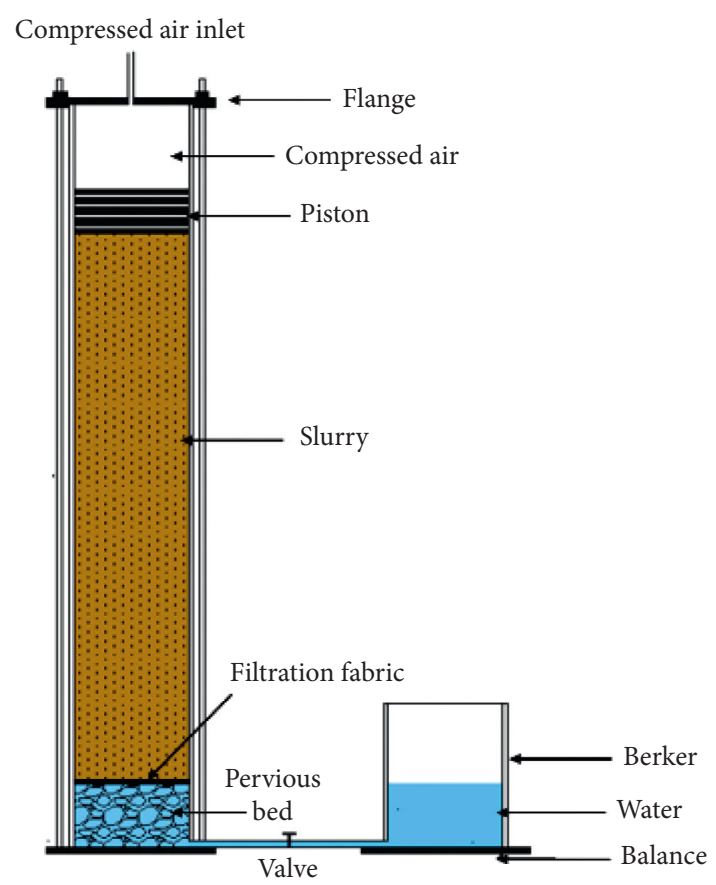

FIgURE 3: Schematic diagram of the slurry filter press.

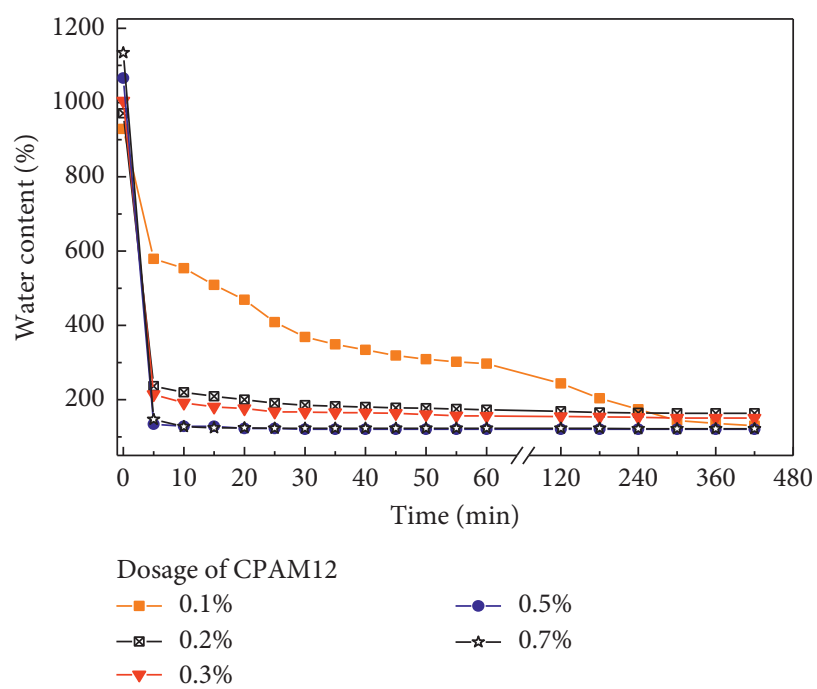

FIGURE 4: Water content curves of sediment with time after adding CPAM12.

slurry decreased by about $360 \%$ at 5 minutes. When more CPAM12 was added, the curve of the slurry water content did not significantly change. Figure 5 shows the SS curve of the slurry supernatant after adding CPAM12. It can be seen that the SS of the slurry supernatant did not meet the Standard for the Pollutant Discharge from Urban Sewage Treatment Plant (GB 18918-2002) (50) when $0.1 \%$ of CPAM12 was added. When the dosage of CPAM12 added was increased to $0.2 \%$ or more, the slurry supernatant met the discharge standard. This also can be seen intuitively from Figure 6. After considering the economic benefits, $0.2 \%$ was chosen as the optimum dosage of CPAM12 to be added. An identical approach was used to select the optimal dosage for 


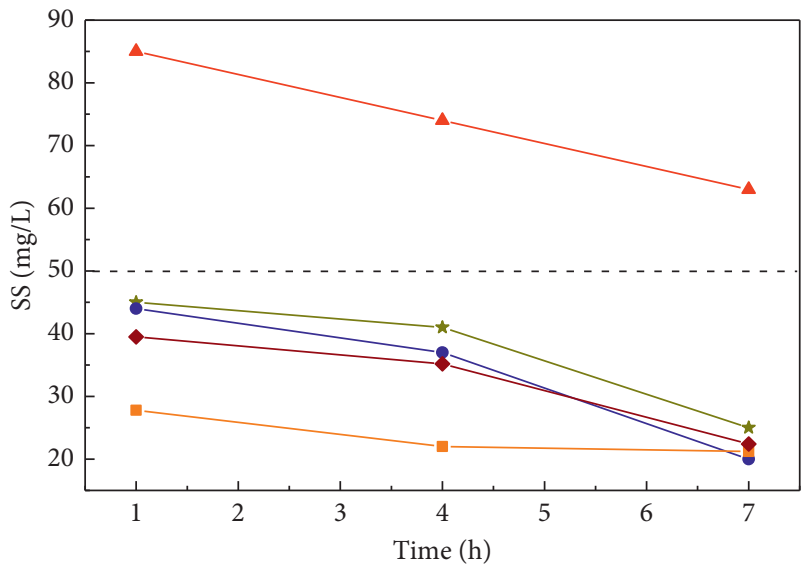

Dosage of CPAM12

$\begin{array}{ll}-\nabla-0.1 \% & -\square-0.5 \% \\ -\star-0.2 \% & -\bullet-0.7 \% \\ -\bullet-0.3 \% & \end{array}$

FIGURE 5: The change curve of SS in supernatant with time after adding CPAM12.

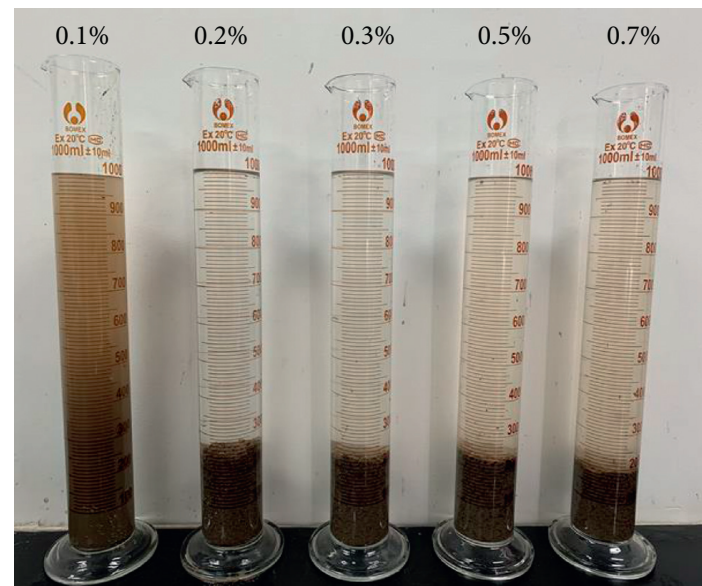

Figure 6: Photo of slurry settlement $1 \mathrm{~h}$ after the addition of CPAM12.

the other 4 flocculants. Table 3 shows the comparison results of flocculant dosages.

Figure 7 shows the variation curve of water content of the sediment with the 5 flocculants applied (using the optimum dosage). It can be seen that the natural settling speed of the original mud was slow, and the water content of the sediment was still as much as $600 \%$ after 7 hours. After adding flocculant and allowing natural settlement for 7 hours, the water content of the sediment was about $100 \%-$ $200 \%$, which is about $400 \%-500 \%$ lower than that of the original slurry. This shows that adding flocculant can significantly increase the flocculation settlement rate of slurry particles and rapidly reduce the water content of slurry.

After adding $\mathrm{FeCl}_{3}$ and $\mathrm{PAC}$, the variation in water content of the sediment was approximately the same, and the water content of slurry after natural settlement for $7 \mathrm{~h}$ was almost the same. After the addition of CPAM3, CPAM12, and APAM12, the settling speed of slurry particles increased.
TABLE 3: Optimum dosage of flocculants.

\begin{tabular}{lc}
\hline Flocculant & Optimum dosage (\%) \\
\hline $\mathrm{FeCl}_{3}$ & 1.6 \\
PAC & 0.8 \\
CPAM3 & 0.3 \\
CPAM12 & 0.2 \\
APAM12 & 0.3 \\
\hline
\end{tabular}

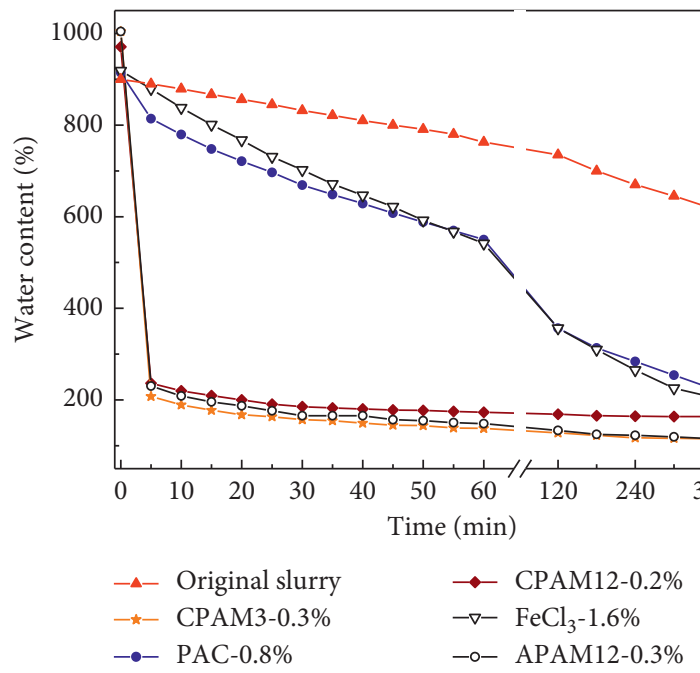

FIgURE 7: Water content curve of sediment using different flocculants.

At 5 minutes, the water content of sediment was about $200 \%$, which is about $600 \sim 700 \%$ lower than that produced by the inorganic flocculants. However, the water content of the slurry after 7 hours of flocculation was little different from that when inorganic flocculants were used. In general, the flocculation effect of organic macromolecule flocculants was better than that of inorganic flocculants. Organic flocculants can realize the rapid separation of slurry and water and facilitate later treatment.

3.2. Analysis of Slurry Particle Size. The distribution of slurry aggregates after adding the five flocculants (optimum dosage) was measured using a laser particle size analyzer. Figure 8 shows the aggregate frequency distribution of slurry with the five flocculants and without a flocculant. It can be seen that the aggregate frequency distribution curve of mud aggregates after adding CPAM3, CPAM12, and APAM12 shifts to the left compared with that of mud without added flocculant. The aggregate content with a particle size of greater than 100 micron in the mud after adding PAM is clearly larger than that of the original mud. The particle size curve for the slurry with inorganic flocculants also shifted to the left, but the range was smaller than that for the organic polymer flocculant. This shows that the flocculant agglomerates the particles in the slurry, and the size of the particles formed in the slurry after adding PAM is larger than that for inorganic flocculant. Thus, the flocculation settling speed of the slurry after adding PAM is the fastest, 


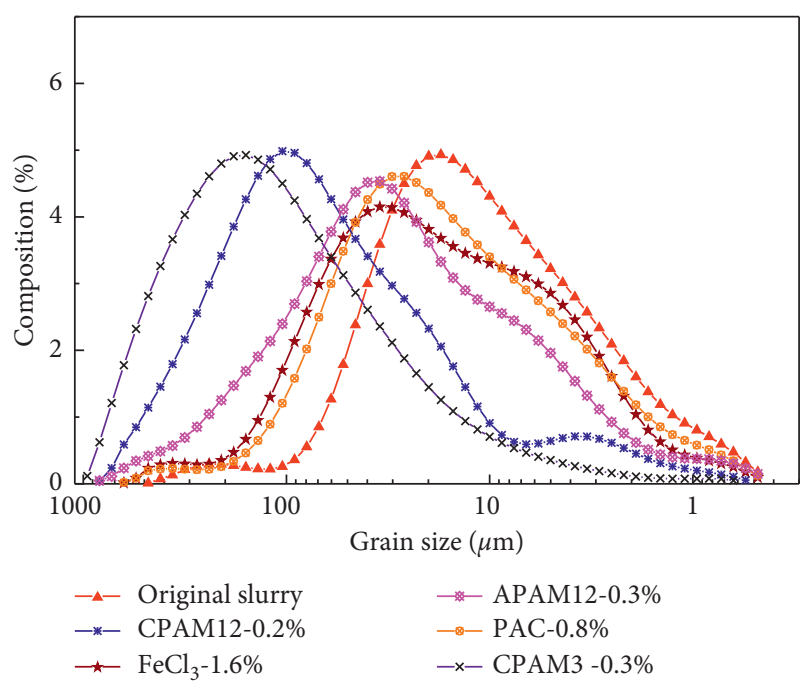

Figure 8: Aggregate frequency distribution of slurry without flocculant and with different flocculants.

followed by the slurry with inorganic flocculant and original slurry. These changes in the settling rate are consistent with the water content change curve for the sediments (Figure 7). It can therefore be concluded that the agglomeration of slurry particles by flocculants is the reason for the rapid flocculation and settling of slurry, and the larger the aggregate size is, the faster the settling speed is.

3.3. Analysis of Slurry Zeta Potential. The zeta potential is an important index that measures the stability of colloids and reflects the ability of colloidal particles to repel or attract each other. The zeta potential of the slurry with flocculant was measured using a laser particle size analyzer. Figure 9 shows the zeta potential curve for the slurry with flocculant. It can be seen that the addition of flocculant caused the slurry zeta potential to change. After adding APAM12 (with a negative charge after hydrolysis), the zeta potential of slurry (generally taken as an absolute value) shows an upward trend, while after adding the other 4 flocculants (with a positive charge after hydrolysis), the zeta potential of slurry shows a downward trend. This indicates that flocculant cations after hydrolysis can repulse cations attracted by negative charges on the surface of soil particles, thus compressing the double layer and reducing the zeta potential. The smaller the zeta potential is, the smaller the repulsion force between particles is and the easier it is to aggregate into clusters [28]. After adding inorganic flocculant, the zeta potential of slurry decreased significantly. This decrease was the main cause of the flocculation settlement of the slurry.

When compared with inorganic flocculants, organic macromolecule flocculants had less influence on the zeta potential of the slurry, and even APAM12 increased the slurry's zeta potential. Therefore, the ability of particles to aggregate when organic flocculant is added is weak. However, after adding PAM, the size of aggregates produced by the slurry is generally larger than that after adding inorganic

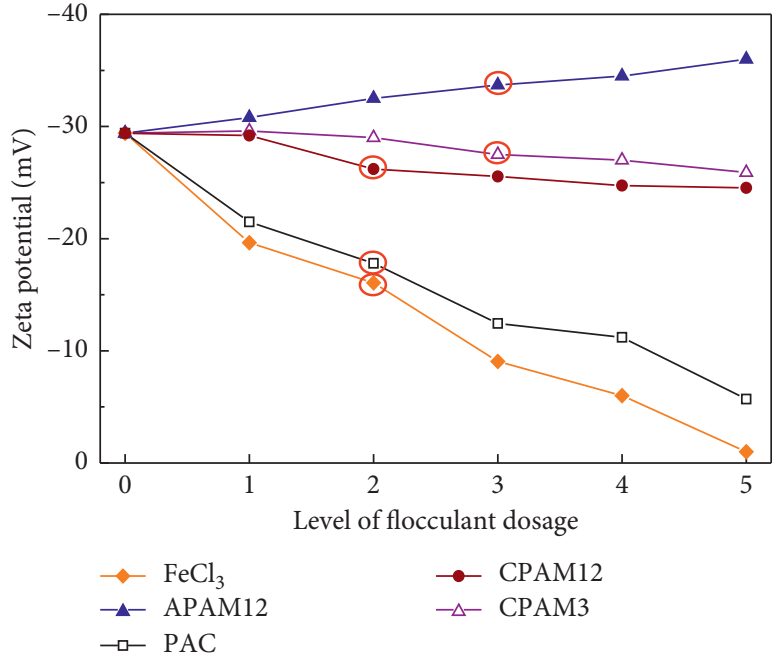

Figure 9: Zeta potential curve of slurry with different flocculant dosages $(1,2,3,4$, and 5 correspond to the different dosages in Table 2, and 0 refers to no flocculant addition. The point marked by red circle refers to the zeta potential corresponding to the optimal dosage of each flocculant).

flocculants (Figure 8). Compared with the reduction in zeta potential, adsorption bridging [29] (see Figure 10) of organic polymer flocculants can more easily agglomerate slurry and play a dominant role in promoting flocculation and settlement of slurry. This explains why the flocculation effect of organic polymer flocculant is better than that of inorganic flocculant.

\section{Results and Discussion of Pressure Filtration Dewatering Test}

4.1. Effect of Different Flocculants on Dewatering Performance of Slurry. Slurry with the different flocculants (optimal dosage) was dewatered by pressure filtration with the device depicted in Figure 3. The pressure was $0.8 \mathrm{MPa}$. If slurry without flocculant is included, a total of 6 groups of experiments were carried out. The water content of slurry at different times was determined from the quality of the filtered water, and a water content curve for the slurry was drawn (Figure 11). Table 4 provides the specific values used in Figure 11. It can be seen that when no flocculant is added, it takes a long time ( 100 minutes) for slurry pressure filtration to reach stability (water content no longer changes with time), and the final water content of the mud cake was $39.1 \%$. When dosed with flocculant, the time required to achieve stability and the final water content of the mud cake are reduced (Table 4). The results show that all five flocculants can improve the pressure filtration dewatering performance of slurry.

When compared with the slurry without flocculant, the time required for slurry pressure filtration to reach stability was decreased by 15 minutes, 30 minutes, and 45 minutes after adding CPAM3, APAM12, and CPAM12, respectively. The final water content of the mud cake was reduced by $10.8 \%, 11 \%$, and $11.2 \%$, respectively. The addition of 


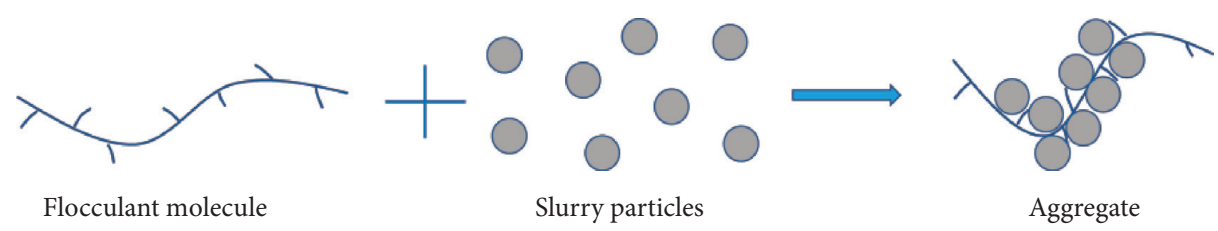

Figure 10: Illustration of the function of flocculant adsorption bridging (adapted from [29]).

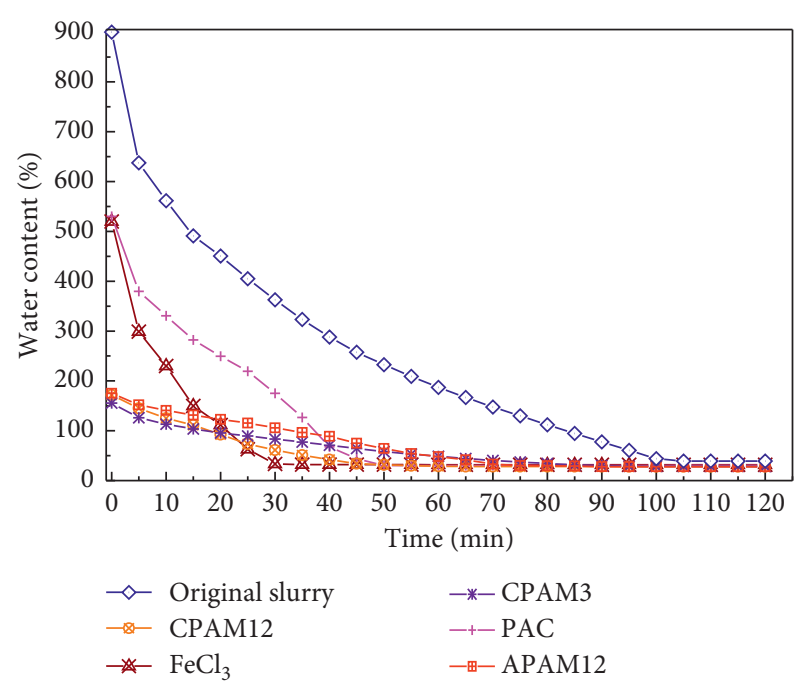

FIGURE 11: Water content change curve for slurry pressure filtration after flocculation.

TABLE 4: Results of pressure filtration test.

\begin{tabular}{lcc}
\hline Flocculant & $\begin{array}{c}\text { Time required for pressure } \\
\text { filtration to reach stability } \\
(\mathrm{min})\end{array}$ & $\begin{array}{c}\text { Final water content } \\
\text { of mud cake }(\%)\end{array}$ \\
\hline- & 100 & 39.1 \\
CPAM3 & 85 & 28.3 \\
APAM12 & 70 & 28.1 \\
CPAM12 & 55 & 27.9 \\
PAC & 50 & 30.4 \\
$\mathrm{FeCl}_{3}$ & 30 & 31.6 \\
\hline
\end{tabular}

CPAM12 resulted in the largest reduction in the time required for slurry pressure filtration to reach stability, and the final water content of the mud cake was reduced the most. This shows that, of the selected PAMs, CPAM12 is the best at improving the dewatering performance of slurry.

When compared with the slurry without flocculant, the time required for slurry pressure filtration to reach stability was decreased by 70 minutes and 50 minutes after adding $\mathrm{FeCl}_{3}$ and PAC, respectively, and the final water content of the mud cake was reduced by $7.5 \%$ and $8.7 \%$, respectively. $\mathrm{FeCl}_{3}$ can improve the dewatering performance of slurry better than PAC. After adding inorganic flocculant, the time needed to stabilize the slurry filter press is lower than that after adding CPAM12, although the water content of the mud cake is slightly higher. In general, although inorganic flocculants have a relatively poor flocculation and settlement effect, they can significantly improve the pressure filtration dewatering performance of the slurry. Finally, considering the flocculation settlement effect of slurry, CPAM12 is considered to be the best to improve slurry treatment efficiency in this study.

4.2. Influence of Different Pressures on Dewatering Effect of Slurry. CPAM12 had better flocculation and dewatering effect in the present study and was selected to investigate the effect of different pressures on the dewatering performance of slurry. The dosage used was $0.2 \%$. Figure 12 shows the curve of the time required for the slurry to reach stability after adding CPAM12 under different pressures, and the change in the final water content of the mud cake. It can be seen that the time to reach stability and the final water content of the mud cake, decreased as the pressure was increased. Thus, increasing the pressure can improve the dewatering effect of the slurry. When the pressure was increased from $0.4 \mathrm{MPa}$ to $0.8 \mathrm{MPa}$, the time required for slurry pressure filtration to reach stability and the final water content of the mud cake, showed a large decrease. When the pressure was increased from $0.8 \mathrm{MPa}$ to $1.2 \mathrm{MPa}$, the final water content of the mud cake was reduced by only $0.6 \%$, and the time required for slurry pressure filtration to reach stability remained unchanged. This shows that a pressure of $0.8 \mathrm{MPa}$ is essentially sufficient to maximize the effect of slurry filtration and that increasing the pressure will not significantly improve slurry filtration.

4.3. Effect of Slurry Particle Size on Dewatering Performance of Slurry. CPAM12 was selected to study the effect of slurry particle size on the dewatering performance of the slurry. As can be seen in Figure 4, when different dosages of CPAM12 were added, differences in the water content curve of the slurry resulted, indicating that the sizes of the aggregates produced in the slurry were also different. A laser particle size analyzer was used to measure the particle size of the slurry after adding different dosages of CPAM12, and a particle size distribution curve was obtained (see Figure 13). It can be seen that, with the increase of CPAM12 dosage, the particle size distribution curve of the slurry shifts to the left when compared with the original slurry. To characterize the aggregate size, D10, D30, and D60 $\left(\mathrm{D}_{x}\right.$ refers to the particle size that the $x \%$ particle size in the slurry was less than this value) were presented in Table 5. When the flocculant dosage was increased, D10, D30, and D60 increased. However, when the dosage was increased to more than $0.3 \%$, the curves were only slightly changed from the $0.3 \%$ dose curve.

The water content of the slurry after the addition of different dosages of CPAM12 and $1 \mathrm{~h}$ of flocculation was adjusted to $190 \%$ by adding water, so as to avoid the possible influence of different initial water contents of slurry on the 


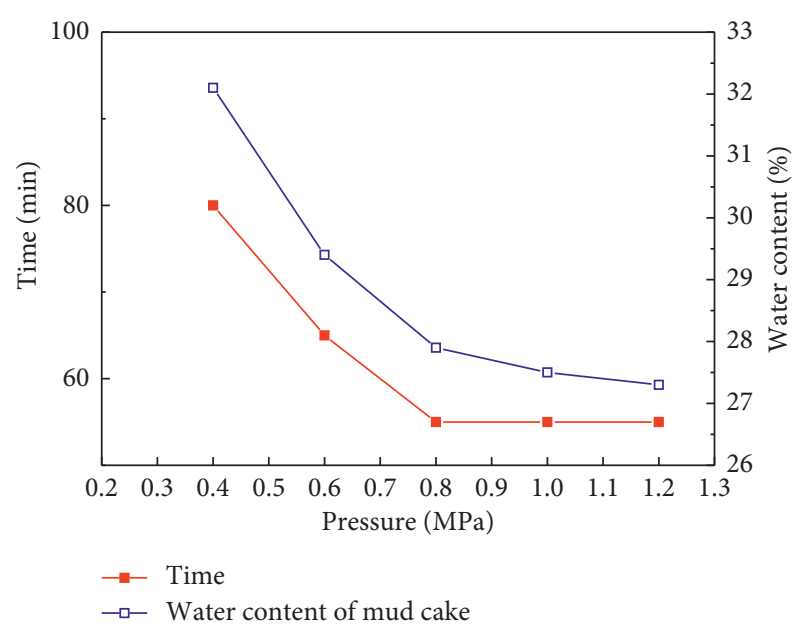

FIGURE 12: Effects of changing pressure on the time required for slurry filtration to reach stability and the final water content of the mud cake, after adding CPAM12.

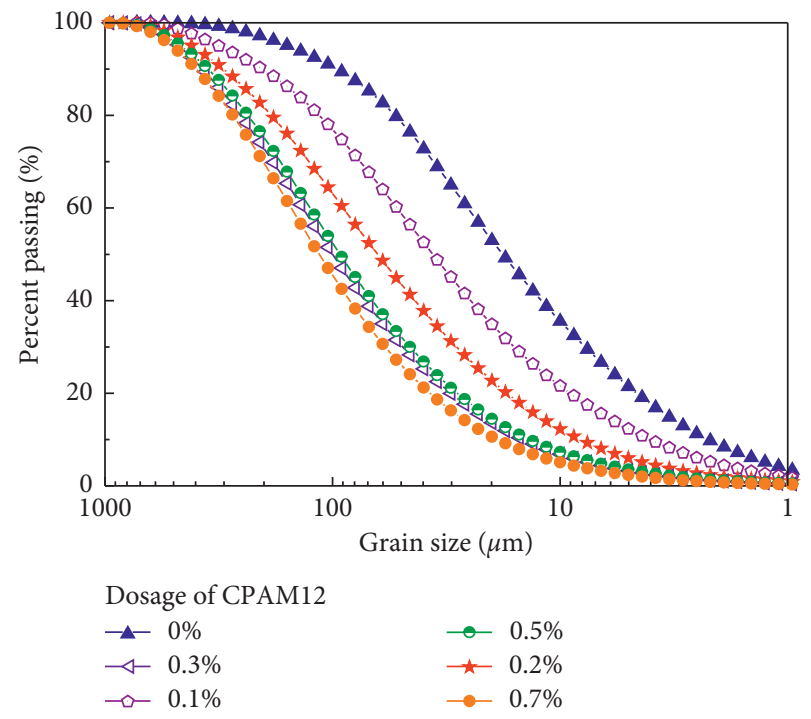

FIGURE 13: Grain size distribution of the slurry with different dosages of CPAM12.

TABLE 5: Summarized measurements of slurry aggregate size after adding different dosages of CPAM12.

\begin{tabular}{lcccc}
\hline Dosage of CPAM12 $(\%)$ & $d_{10}(\mu \mathrm{m})$ & $d_{30}(\mu \mathrm{m})$ & $d_{60}(\mu \mathrm{m})$ & $C_{u}$ \\
\hline 0 & 2.21 & 7.72 & 26.2 & 11.85 \\
0.1 & 4.4 & 16.8 & 50.5 & 11.48 \\
0.2 & 8.85 & 29.3 & 89.5 & 10.11 \\
0.3 & 13.32 & 46.5 & 127.1 & 9.54 \\
0.5 & 14.41 & 51.3 & 134.2 & 9.31 \\
0.7 & 15.1 & 53.2 & 138.1 & 9.14 \\
\hline
\end{tabular}

Note. $C_{u}=\left(d_{60} / d_{10}\right)$ is the nonuniformity coefficient used to evaluate the gradation of soil particles.

dewatering performance of slurry. The device depicted in Figure 3 was used to test the effect of slurry pressure filtration. The pressure was $0.8 \mathrm{MPa}$. A water content change curve was compiled (Figure 14). It can be seen that when the
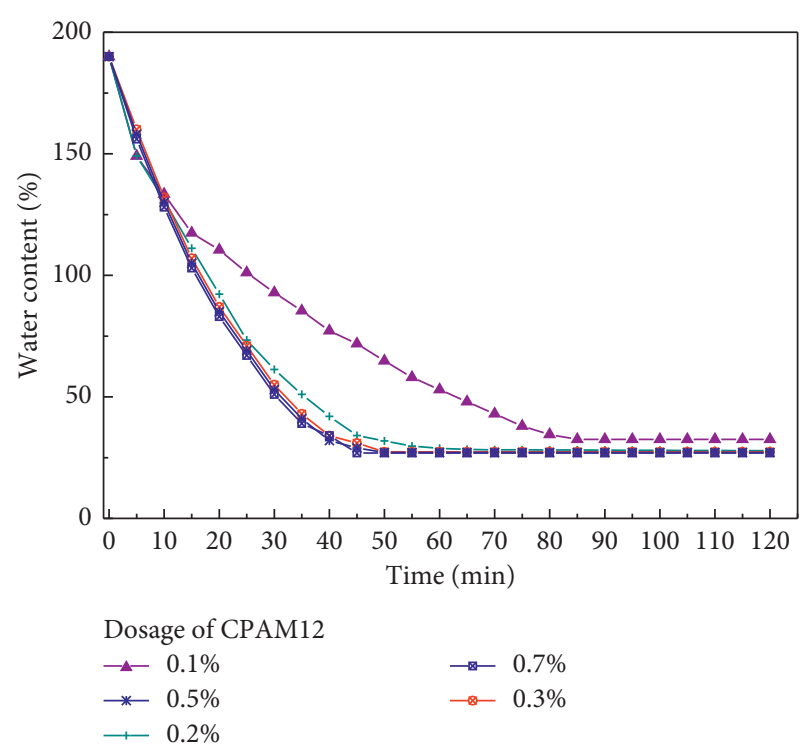

FIGURE 14: Water content change curve of slurry pressure filtration with different CPAM12 dosages.

dosage is $0.1 \%$, it takes a long time ( 85 minutes) for the slurry pressure filtration to reach stability. When the dosage was increased to $0.2 \%$, the time required for the slurry to reach pressure filtration stability was 55 minutes. This is significantly shorter than that for the $0.1 \%$ dosage, and the water content was also reduced. When the dosage was $0.3 \%$, the time required for the slurry pressure filtration to reach stability was 50 minutes, only 5 minutes less than that of $0.2 \%$ dosage, and the water content was little changed from the $0.2 \%$ dosage. Further increases in dosage did not cause significant reductions in the time required for the slurry pressure filtration to reach stability and in the final water content of the mud cake.

To further explore the relationship between particle size and the dewatering performance of slurry, variation curves for stability time and the nonuniformity coefficient of slurry pressure filtration were compiled (Figure 15). It can be seen that, with the increase in flocculant dosage, the nonuniformity coefficient decreases gradually, as does the time required for pressure filtration to reach stability. In soil mechanics, the larger the nonuniformity coefficient of soil particle is, the better the particle gradation is. The soil contains sufficient small particles to fill the pore spaces formed by large particles, and the soil is easily compacted. Conversely, soil with a small nonuniformity coefficient is not easily compacted. In the present study, the time required for the stabilization of slurry pressure filtration decreased with the decrease in slurry particle size distribution, which indicates that the behavior of the high water content slurry can be explained in terms of the nonuniformity coefficient. The larger the nonuniformity coefficient is, the easier it is for the fine particles in the slurry to fill the pore spaces formed by large particles. This reduces the pore size of the slurry particles and blocks the drainage channels, thereby reducing the drainage rate. At the macroscopic level, it takes a long time for slurry filtration to reach stability. 


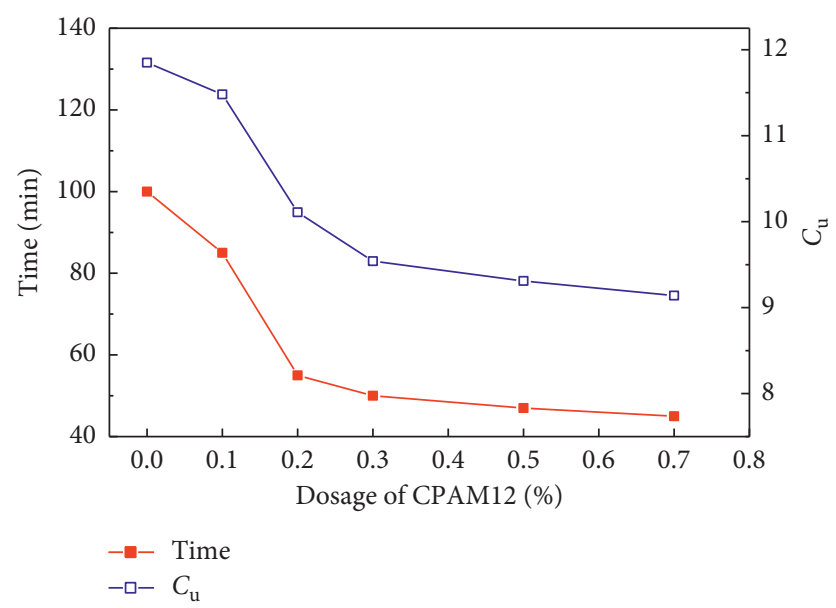

FIGURE 15: Change curves of the time required for pressure filtration to reach stability and of the nonuniformity coefficient for different dosages of CPAM12.

Compared with the organic polymer flocculant, the inorganic flocculant can make the slurry form aggregates with smaller particle size and the flocculation settling effect is poor, but inorganic flocculants can significantly improve the pressure filtration dewatering performance of the slurry (Table 4). CPAM3, CPAM12, and APAM12 can all make the slurry flocculate and settle rapidly, but only CPAM12 can significantly improve the dewatering performance of the slurry (Table 4). To determine whether these differences are related to the gradation of the slurry particle after flocculation, the distribution of aggregates in the slurry after adding inorganic and organic flocculants at the optimum dosage was measured (see Table 6). The results were combined with data from Table 4 , and the variation curves of the nonuniformity coefficient and the time required for the stability of the slurry after adding different flocculants were compiled (Figure 16). It can be seen that the time required for pressure filtration stabilization decreased with the decrease in the nonuniformity coefficient. The addition of the inorganic flocculant $\mathrm{FeCl}_{3}$ resulted in the lowest nonuniformity coefficient for the slurry. The addition of PAC resulted in the second-lowest nonuniformity coefficient. After the addition of CPAM12, the nonuniformity coefficient of the slurry is lower than that of the slurry after the addition of CPAM3 and APAM12. This also shows that flocculants improve the pressure filtration dewatering performance of slurry by making the particle size distribution more uniform, and the smaller the nonuniformity coefficient is, the better the pressure filtration dewatering performance of the slurry is.

Because flocculants can agglomerate slurry particles into aggregates and change their gradation, flocculants can improve the dewatering performance of slurry. In addition, the different dosages can also affect the nonuniformity coefficient of slurry. Flocculant and dosage can therefore be selected by testing the nonuniformity coefficient of the flocculated slurry. This will enable the dewatering effect to be predicted and thereby improve the slurry treatment efficiency.
TABLE 6: Summary of measurements of slurry aggregate size after adding different flocculants.

\begin{tabular}{lcccc}
\hline Flocculant & Dosage $(\%)$ & $d_{10}(\mu \mathrm{m})$ & $d_{60}(\mu \mathrm{m})$ & $C_{u}$ \\
\hline- & - & 2.21 & 26.2 & 11.85 \\
CPAM3 & 0.3 & 11.5 & 131.1 & 11.4 \\
APAM12 & 0.3 & 4.8 & 51.9 & 10.81 \\
CPAM12 & 0.2 & 8.85 & 89.5 & 10.11 \\
PAC & 0.8 & 3.3 & 31.2 & 9.45 \\
$\mathrm{FeCl}_{3}$ & 1.6 & 3.8 & 33.4 & 8.78 \\
\hline
\end{tabular}

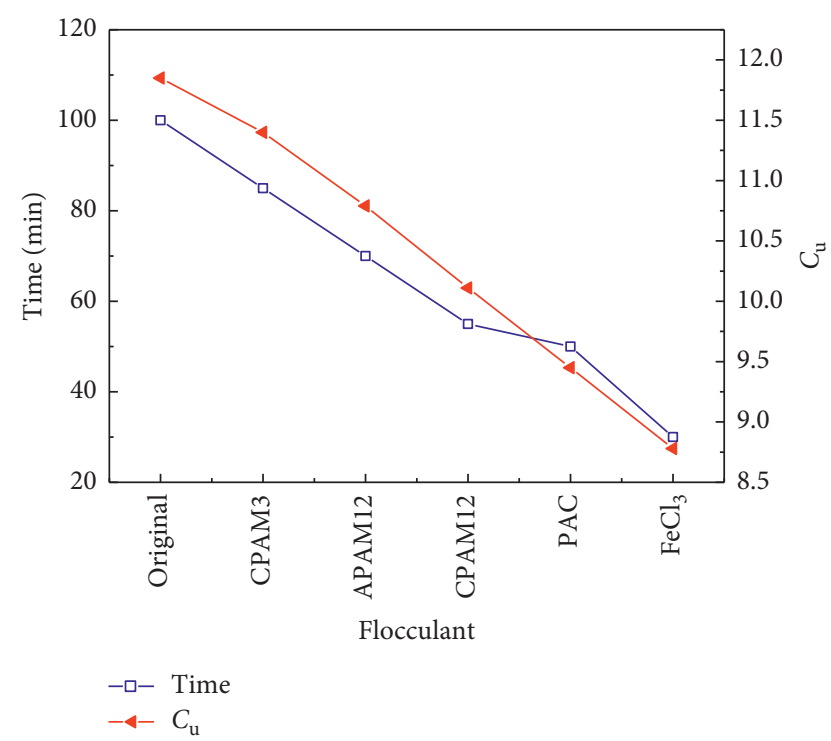

FIGURE 16: Change curve of the time required for pressure filtration to reach stability and of the nonuniformity coefficient for different flocculation treatments (original refers to slurry without adding flocculant).

\section{Concluding Remarks and Future Research}

In this study, a flocculation settling test on waste slurry was carried out using various inorganic and organic flocculants, and the changes in the properties of the slurry after flocculation and the effect of pressure filtration dewatering were tested. Ultimately, the influence of the change of the basic properties of the slurry on flocculation settlement and pressure filtration of the slurry was analyzed. This study has yielded the following conclusions:

(1) The addition of flocculant makes the slurry particles form aggregates, which led to rapid precipitation of the slurry. Inorganic flocculants significantly reduced the zeta potential value of the slurry. Although organic macromolecule flocculants had little effect on the zeta potential, they can produce larger size aggregates, thereby resulting in a better flocculation effect than inorganic flocculants.

(2) Inorganic and organic flocculants can improve the dewatering performance of slurry. Of the flocculants for pressure filtration dewatering test, $\mathrm{PAC}, \mathrm{FeCl}_{3}$, and CPAM12 gave the better results. Considering the flocculation settlement effect of slurry, CPAM12 is 
considered to be the best to improve slurry treatment efficiency. With increased pressure, the dewatering effect on the slurry gradually improves, until it reaches a certain value. Subsequently, increases in pressure do not lead to further improvements in the dewatering of the slurry.

(3) Different flocculants and different dosages change the nonuniformity coefficient of the slurry. Change in the nonuniformity coefficient is the main cause of improvement of pressure filtration dewatering performance of the slurry. Through testing the nonuniformity coefficient of flocculated slurry, the optimal flocculant can be selected and the optimum dosage can be determined. This allows the dewatering effect to be predicted, thereby improving the slurry treatment efficiency.

(4) In the future, a microscopic test, such as test on the pore characteristics of the mud cake, is needed to be performed to further verify the mechanism of flocculants improving the dewatering performance of slurry.

\section{Data Availability}

The data used to support the findings of this study are available from the corresponding author upon request.

\section{Conflicts of Interest}

The authors declare that they have no conflicts of interest.

\section{Acknowledgments}

The authors gratefully acknowledge the financial support from the National Natural Science Foundation of China (nos. 51778213 and 52078189) and Fundamental Research Funds for the Central Universities of China (no. B200202073).

\section{References}

[1] F. L. Min, W. Zhu, and X. R. Han, "Filter cake formation for slurry shield tunneling in highly permeable sand," Tunnelling and Underground Space Technology, vol. 38, no. 3, pp. 423430, 2013.

[2] P. H. Sun, B. K. Zhao, H. Cao et al., "Lab study on the effect of cation exchange capacity on slurry performance in slurry shields," Advances in Civil Engineering, vol. 2018, Article ID 2942576, , 2018.

[3] X. B. Li, R. Zhao, H. T. Fu et al., "Slurry improvement by vacuum preloading and electro-osmosis," Proceedings of the Institution of Civil Engineers - Geotechnical Engineering, vol. 172, no. 2, pp. 145-154, 2019.

[4] T. W. Yee, C. R. Lawson, Z. Y. Wang, L. Ding, and Y. Liu, "Geotextile tube dewatering of contaminated sediments, Tianjin Eco-City, China," Geotextiles \& Geomembranes, vol. 31, no. 4, pp. 39-50, 2011.

[5] H. Mori, H. Miki, and N. Tsuneoka, "The geo-tube method for dioxin-contaminated soil," Geotextiles and Geomembranes, vol. 20, no. 5, pp. 281-288, 2002.
[6] C. R. Lawson, "Geotextile containment for hydraulic and environmental engineering," Geosynthetics International, vol. 15, no. 6, pp. 384-427, 2008.

[7] Y. N. Yu and Y. P. Zhang, "Test study on engineering properties of dredging soil of West lake in Hangzhou," Rock and Soil Mechanics, vol. 25, no. 4, pp. 579-582, 2004.

[8] Y. J. Wu, K. Niu, Y. T. Lu et al., "Anti-clogging mechanism of vacuum preloading with flocculation in treating construction waste slurry," Chinese Journal of Geotechnical Engineering, vol. 50, no. 6, pp. 95-103, 2017.

[9] R. Li, X. H. Zhao, Y. B. Cong, Q. Hu, and Y. Gao, "Experimental study on environmental dredging of a city river," Water Resources and Hydropower Engineering, vol. 35, no. 5, pp. 19-21, 2004.

[10] S. Bourgès-Gastaud, G. Stoltz, F. Sidjui, and N. Touze-Foltz, "Nonwoven geotextiles to filter clayey sludge: an experimental study," Geotextiles and Geomembranes, vol. 42, no. 3, pp. 214-223, 2014.

[11] G. Viccione, S. Evangelista, A. Armenante, and V. Ricciardi, "Clogging process and related pressure drops in wire-wound filters: laboratory evidence," Environmental Science and Pollution Research, vol. 27, no. 3, pp. 23464-23476, 2020.

[12] S. Evangelista, G. Viccione, and O. Siani, "A new cost effective, long life and low resistance filter cartridge for water treatment," Journal of Water Process Engineering, vol. 27, pp. 1-14, 2019.

[13] Y. Zhou, Y. F. Gao, H. Tao, and Y. J. Chai, "Drainage-Promotion effect of aeration vacuum rapid slurry-water separating techinique on dredged sludge," Chinese Journal of Rock Mechanics and Engineering, vol. 29, no. 1, pp. 3064-3070, 2010.

[14] A. Ding, F. S. Qu, H. Liang et al., "Effect of adding wood chips on sewage sludge dewatering in a pilot-scale plate-and-frame filter press process," RSC Advances, vol. 4, no. 47, pp. 24762-24768, 2014.

[15] J. T. Novak, "Dewatering of sewage sludge," Drying Technology, vol. 24, no. 10, pp. 1257-1262, 2006.

[16] S. Q. Ou and M. M. Jiang, "Research progress of polymer sludge dewatering flocculants," Guangdong Chemical Industry, vol. 45, no. 7, pp. 166-168, 2018.

[17] L. J. Guo, S. D. Li, and C. Li, "The study of effect of flocculants to municipal sludge dewatering Performance," Shandong Chemical Industry, vol. 46, no. 22, pp. 161-162, 2017.

[18] S. K. Bhatia and R. Satyamurthy, "Effect of polymer conditioning on dewatering characteristics of fine sediment slurry using geotextiles," Geosynthetics International, vol. 16, no. 2, pp. 83-96, 2009.

[19] L. D. Suits, T. C. Sheahan, R. M. Koerner, and G. R. Koerner, "Performance tests for the selection of fabrics and additives when used as geotextile bags, containers, and tubes," Geotechnical Testing Journal, vol. 33, no. 3, pp. 236-242, 2010.

[20] R. A. Bader, S. K. Bhatia, M. M. Khachan, D. Cetin, and B. V. Ramarao, "Cationic starch flocculants as an alternative to synthetic polymers in geotextile tube dewatering," Geosynthetics International, vol. 21, no. 2, pp. 119-136, 2014.

[21] B. W. Maurer, A. C. Gustafson, S. K. Bhatia, and A. M. Palomino, "Geotextile dewatering of flocculated, fiber reinforced fly-ash slurry," Fuel, vol. 97, no. 7, pp. 411-417, 2012.

[22] J. W. Worley, T. M. Bass, and P. F. Vendrell, "Use of geotextile tubes with chemical amendments to dewater dairy lagoon solids," Bioresource Technology, vol. 99, no. 10, pp. 4451-4459, 2018.

[23] X. Li, "Flocculation and dewatering experimental study of waste slurry in slurry shield," Railway Engineering, vol. 58, no. 5, pp. 144-147, 2018. 
[24] C. L. Zuo, "Experimental study on rapid separation between water and slurry from bored pile," Construction Technology, vol. 47, no. 1, pp. 66-69, 2018.

[25] Q. X. Zhang, T. Tao, X. J. Wang, and Y. X. Zhai, "Experimental study on treatment of waste slurry in cast-in-situ bored pile," Journal of Hydraulic Engineering, vol. 46, no. 1, pp. 40-45, 2015.

[26] S. L. Wu, W. Zhu, F. L. Min, and X. H. Fan, "A test method for measuring floc size of slurry," Geotechnical Testing Journal, vol. 41, no. 6, pp. 998-1007, 2018.

[27] S. L. Wu, W. Zhu, F. L. Min et al., "Clogging mechanism and effect of cake permeability in soil-water separation using vacuum filtration," Chinese Journal of Geotechnical Engineering, vol. 39, no. 8, pp. 1530-1537, 2017.

[28] F. L. Min, J. R. Du, N. Zhang et al., "Experimental study on property change of slurry and filter cake of slurry shield under seawater intrusion," Tunnelling and Underground Space Technology, vol. 88, pp. 290-299, 2019.

[29] J. He, J. Chu, S. K. Tan, T. T. Vu, and K. P. Lam, "Sedimentation behavior of flocculant-treated soil slurryflocculanttreated soil slurry," Marine Georesources \& Geotechnology, vol. 35, no. 5, pp. 593-602, 2017. 